\title{
Sports-related injuries in elderly men still active in sports
}

\author{
M. Kallinen, $\mathrm{MD}^{* \dagger}$, M. Alén, MD PhD ${ }^{\dagger}$ \\ ${ }^{*}$ LIKES-Research Centre for Physical Culture and Health, Jyväskylä, Finland, and ${ }^{\dagger}$ Peurunka-Rehabilitation and \\ Physical Exercise Centre, Laukaa, Finland
}

\begin{abstract}
By means of a questionnaire with a complementary interview and physical examination, the site and nature of sports injuries were investigated over a 10-year period (1977-1987) in 97 elderly athletes (age range 70-81 years). The athletes were still active in training and competition with a mean competition background of 15 years. Of the subjects studied 30 were strength/power athletes and the remaining 67 endurance athletes. Altogether we found 273 sports-related injuries (169 acute and 104 overuse injuries). Of the injuries $75 \%$ had occurred in the lower extremities. The most commonly injured part of the body was the knee ( $20 \%$ of all cases). Sprains of the thigh and knee were the most frequent types of acute injury. In most cases the treatment prescribed was rest and physiotherapy. Surgery had been necessary in ten cases $(3.7 \%$ of all injuries). Mean withdrawal from normal sporting activity had, in general, been 2-3 weeks. Of the injuries, one in five had, however, lasted over several years causing some disability during sporting activities.
\end{abstract}

Keywords: Sports injuries, elderly men, physically active

It is well known that physically active children commonly sustain sports-related overuse injuries associated with the growth and development of their musculoskeletal system. The proportion of acute sports-related injuries seems also to be high among prepubertal children ${ }^{1}$. In adult athletes a greater variation in the pattern of injury in relation to the changing nature of sports with age has been reported. Among ageing athletes the incidence of inflammatory overuse injuries is higher than acute injuries ${ }^{2,3}$.

Ageing causes many structural and functional changes which may increase the risk of injuries. On the other hand, since ageing also retards healing, elderly athletes would be expected to sustain a greater proportion of the injuries associated with the ageing process itself ${ }^{4}$. There is, however, limited data on the sports-related injuries prevalent among competitively active elderly athletes aged 70 years and over.

The purpose of this study was to describe the frequency, mechanisms and nature of sports-related

Address for correspondence: M. Kallinen, Peurunka-Rehabilitation and Physical Exercise Centre, SF-41350 Laukaa as., Finland

(C) 1994 Butterworth-Heinemann Ltd

0306-3674/94/010052-04 injuries in elderly men (aged over 70 years) still active in competitive sports. To elucidate the effect of sports event and type of training on the nature of injuries, strength/power and endurance trained athletes were compared.

\section{Materials and methods}

The study was performed as part of a larger research project on health and functional capacity in elderly athletes and sedentary men. The initial group of subjects consisted of 287 elderly men athletes drawn from a register of Finnish sport organizations. Of this number, 220 responded to the first questionnaire. The most active athletes (112) were selected for further study. The second questionnaire was then mailed to the 112 selected athletes and drew a response rate of $88 \%$ (99 of 112). Of these subjects $98 \%(n=97)$ came to the laboratory for measurements. Thus, the final study group comprised 97 competitively active men (age range 70-81 years). The subjects were divided into two subgroups on the basis of their sport events: endurance $(n=69)$ and strength/power $(n=28)$ athletes (Table 1). The subjects all had a life-long training history (range 31-68 years) and all were still active in competitive sports.

The second mailed questionnaire elicited information about each subject's training and competition background, injury dates during the past 10-year

Table 1. Sports event distribution of 97 elderly athletes aged 70-81 years

\begin{tabular}{lc}
\hline Sports event & Frequency $(\%)$ \\
\hline Strength/power athletes $(\mathbf{n}=28)$ & \\
$\quad$ Track and field, sprinters/jumpers & 13 \\
Track and field, throwers & 12 \\
Other (bowler 1, power lifter 1, tennis-player 1$)$ & 3 \\
Total & 28 \\
Endurance athletes $(\mathbf{n}=69)$ & \\
Cross-country skiers & 28 \\
Orienteers & 25 \\
Endurance runners & 1 \\
Other (walkers 2, cyclist 1$)$ & 3 \\
Total & 72 \\
Overall total & 100 \\
\hline
\end{tabular}


Table 2. Physical characteristics, competition background and training volume (hours during preceding week) in 70-81-year-old strength/power athletes $(n=28)$ and endurance athletes $(n=69)$

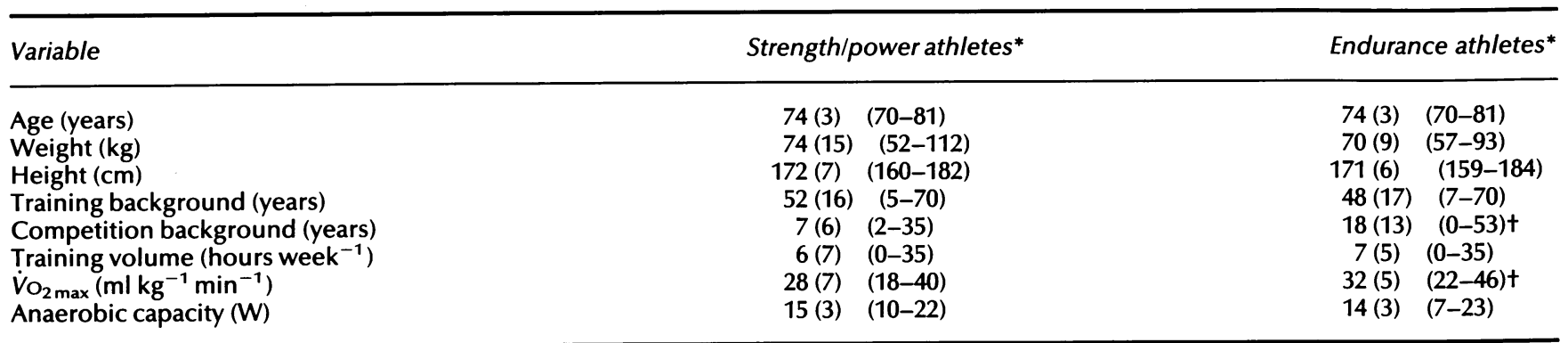

*Values are mean(s.d.) (range); tsignificant $(P<0.05)$ differences between the groups; Student's $t$ test

period, and the mechanism and nature of injuries together with contributory factors, treatment, healing time and resultant permanent disabilities. When the subjects entered the laboratory they were all interviewed by the same physician about their sportsrelated injuries and other past medical problems, on the basis of their responses to the second questionnaire which thus was checked and completed. Special attention during the physical examination was paid to musculoskeletal signs of complications relating to current and/or past injuries. The laboratory investigations consisted of various anthropometric, physical and physiological measurements. Cardiorespiratory capacity was assessed by an ergometer test directly measuring oxygen uptake 5 . Anaerobic power was measured by a jumping test on a contact mat ${ }^{6}$.

All the athletes gave their written informed consent and the Ethical Committee of the University approved the study protocol. Statistical analysis consisted of the $\chi^{2}$ test and Student's $t$ test (two-tailed) evaluations for independent samples. The non-parametric Wilcoxon matched-pairs signedranks test and Mann-Whittney $U$ test were also used $^{7}$.

\section{Results}

The physical and the training characteristics of the subjects are described in Table 2. The endurance athletes had a longer competition background and higher maximal oxygen uptake than the strength/ power athletes.

Of the athletes $81 \%$ had had at least one sports injury. Altogether, 273 sports-related injuries were reported during the 10-year period (1977-1987) of which $62 \%$ (169) were acute and the remaining $38 \%$ (104) were overuse injuries.

The mechanisms of acute injuries are described in Table 3. There were significantly more falls/slips among the endurance athletes than among the strength/power athletes for whom sprains were more common $(85 \%)$.

The anatomical distribution of all injuries is represented in Table 4 . Only $13 \%$ of all injuries were located in the upper extremities, while $75 \%$ were sustained in the lower extremities. The most frequently injured body site was the knee (20\%). Ankle and foot injuries represented $19 \%$ of all injuries. The frequency of overuse injuries in the upper extremities was higher $(P<0.05)$ among strength/power athletes than endurance athletes. Acute injuries, on the other hand, were located significantly more often in the lower extremities among the strength/power athletes than among endurance athletes $(91 \%$ and $59 \%$, respectively). The endurance athletes had, however, significantly more acute upper extremity injuries than the strength/power athletes.

Among both study groups for both types of injury, muscle was the most commonly injured tissue. Injuries to tendons and joints were also quite

Table 3. Mechanisms of acute injuries $(n=159)$

\begin{tabular}{lccc}
\hline Mechanism & \multicolumn{3}{c}{ Frequency of acute injuries } \\
\cline { 2 - 4 } & $\begin{array}{c}\text { All athletes } \\
\text { (\%) }\end{array}$ & $\begin{array}{c}\text { Endurance } \\
\text { athletes (\%) }\end{array}$ & $\begin{array}{c}\text { Strength/power } \\
\text { athletes (\%) }\end{array}$ \\
\hline Fall/slip & 35 & 50 & $12^{*}$ \\
Sprain & 57 & 39 & $85 \dagger$ \\
Traffic accident & 3 & 4 & 2 \\
Other & 5 & 7 & 1 \\
Total & 100 & 100 & 100 \\
\hline
\end{tabular}

Statistical significance: ${ }^{*}(P<0.05), t(P<0.01)$ between the groups; Student's $t$ test

Table 4. Anatomical distribution of all injuries $(n=273)$ in elderly athletes $(n=97)$

Anatomical site of injury

Frequency (\%)

Head and neck

Back

2

Upper extremity

Shoulder

Antebrachium

Fingers

13

Lower extremity

Groin

Hip

Thigh

Knee

Leg

Foot and ankle 
Table 5. Tissue distribution of all sports-related injuries $(n=273)$ in endurance and strength/power athletes

\begin{tabular}{lrr}
\hline Injured tissue & \multicolumn{2}{c}{ Frequency of injuries } \\
\cline { 2 - 3 } & $\begin{array}{c}\text { Endurance } \\
\text { athletes }\end{array}$ & $\begin{array}{c}\text { Strength/power } \\
\text { athletest (\%) }\end{array}$ \\
\hline Muscle & 27 & 46 \\
Tendon & 6 & 10 \\
Tendon insertion & 3 & 14 \\
Bone & 5 & 1 \\
Joint & 28 & 12 \\
Fascia & 0 & 1 \\
Nerve & 5 & 0 \\
Skin & 3 & 3 \\
Bursa & 5 & 1 \\
Others (nails etc.) & 1 & 2 \\
Unspecified & 17 & 10 \\
Total & 100 & 100 \\
\hline
\end{tabular}

${ }^{*}$ No. injuries $=177 ;+$ No. injuries $=96$

common (Table 5). Muscle injuries were, however, more common $(P<0.01)$ among acute injuries than overuse injuries (44\% as against $16 \%$ respectively). Also a significantly higher proportion $(P<0.05)$ of acute injuries occurred in the joints ( $29 \%$ versus $13 \%)$. Of overuse injuries $34 \%$ could not be located in any specific tissue, for example diffuse pain in the leg. Bone fractures represented $5 \%$ of acute injuries but there was not a single stress fracture among the overuse injuries.

The most widely used treatments were rest, physiotherapy and oral drug treatment. Surgery was needed in $2 \%$ of the acute and in $7 \%$ of the overuse injuries. A physician was involved in treating acute injuries $(64 \%)$ significantly more often than overuse injuries $(48 \%)$. There was, however, no difference between the endurance and strength/power athletes in the participation of physicians in treatments.

Sixteen percent of the overuse and $15 \%$ of the acute injuries healed within 1 week. Almost one-fifth of the symptoms of overuse and acute injuries lasted for several years. Healing time did not differ significantly between overuse and acute injuries. There were generally no significant differences in injury healing time between the study groups.

Permanent disability in training and competition occurred in $33 \%$ of overuse injuries and $27 \%$ of acute injuries. Activities associated with daily life, excluding sport, were disturbed by a permanent disability caused by overuse injuries in $9 \%$ of cases and by acute injuries in $6 \%$ of cases.

\section{Discussion}

We asked the elderly athletes to list all injuries sustained during the preceding 10-year period. These athletes were selected citizens - their functional capacity was in excess of that of nonathletes in the same age group. Since they kept detailed training diaries it was possible to obtain a fairly complete account of their injuries. We also used a multiphased study protocol to verify responses to the questionnaires and, by means of a clinical examination, to identify signs of current and past musculoskeletal problems.

Of the athletes $81 \%$ had experienced a sports injury of some kind. The remaining $19 \%$ had sustained no sports-related injuries during the 10-year period. In agreement with our study, Kavanagh and Shephard ${ }^{8}$ reported in 1977 that during the preceding year more than half of a sample of veteran track competitors had sustained an injury which prevented them from training for at least 1 week.

However, in contrast to earlier studies ${ }^{2,3}$ of sports-related injuries among elderly athletes, we found the frequency of acute injuries to be higher than that of overuse injuries. The sports event distribution of our subjects might have contributed to the higher frequency of acute injuries. Of our subjects $53 \%$ were cross-country skiers or orienteers who train and compete on rough terrain which may increase the risk of acute injuries (sprains, strains etc.). This would also seem to explain why the mechanisms of acute injury among endurance athletes (orienteers, cross-country skiers) included more falls and slips. Korpi et al. ${ }^{9}$ found that athletes aged over 50 years, who participated in the orienteering event, visited the first-aid station twice as often as their younger counterparts. Some authors have collected data from the patient registers of relatively large sports polyclinics and found a higher number of overuse injuries among elderly athletes ${ }^{3}$. We believe that this difference may be due to the method of data collection. Most of the acute injuries may have been treated in local health centres, and consequently will not appear in the registers of sports polyclinics. It is also likely that long-lasting injuries (overuse injuries) will accumulate in centres specializing in the treatment of sports injuries.

The number of injuries to the lower extremities seems as high as expected. Also the high frequency of the injuries to joints and muscles is not surprising. Comparing the injury pattern of the two study groups we find that acute injuries seem to be related to falls and slips during skiing and orienteering. This may be due to the greater risks and/or the nature of skills (coordination, balance, reaction time) involved in these sports events.

Most injuries were not protracted and were treated by rest and physiotherapy. The number of injuries treated surgically was low. In general, injuries were not serious. We must, however, remember that it is possible that athletes with more serious injuries have terminated their athletic careers and thus do not appear in the database of this study. There were few differences between the strength/power athletes and endurance athletes in the nature of injuries and they were based mainly on the type of sports event. The frequencies of acute and overuse injuries did not, however, differ between the study groups. Although there were no serious injuries in this study, the overall duration of some injuries causing some disability during training and competition seems rather long. 
The best treatment for sports injuries generally is prevention. Careful warming up before physical exertion is particularly important. Also better protection against falls by improved control over movement and, perhaps, balance training could help in injury prevention. Quick and active treatment of injuries is also important among elderly athletes as ageing tissue has a lower healing capacity. In this way we can diminish the complications and duration of injuries and make sport more enjoyable and safer for elderly people.

\section{References}

1 Jacobsson B. Sports accidents among children and teenagers. Scand J Sports Sci 1986; 8: 75-9.
2 DeHaven KE, Lintner DM. Athletic injuries: comparison by age, sport, and gender. Am J Sports Med 1986; 14: 218-24.

3 Kannus P, Niittymäki S, Järvinen M, Lehto M. Sports injuries in elderly athletes: a three year prospective, controlled study. Age Ageing 1989; 4: 263-70.

4 Menard D, Stanish WD. The aging athlete. Am J Sports Med 1989; 17: 187-96.

5 Rusko H, Rahkila P, Karvinen E. Anaerobic threshold, skeletal muscle enzymes and fiber composition in young cross country skiers. Acta Physiol Scand 1980; 108: 263-8.

6 Bosco C, Luhtanen P, Komi PV. A simple method for measurement of mechanical power in jumping. Europ J Appl Physiol 1983; 51: 129-35.

7 Siegel S. Nonparametric Statistics for the Behavioral Sciences. New York, USA: McGraw-Hill Book Company 1956: 312.

8 Kavanagh T, Shepherd RJ. The effects of continued training on the ageing process. Ann NY Acad Sci 1977; 301: 656-70.

9 Korpi J, Haapanen A, Svahn T. Frequency, location, and types of orienteering injuries. Scand J Sports Sci 1988; 9: 53-6.

\section{TH BIENNIAL CONFERENCE INTERNATIONAL SOCIETY FOR COMPARATIVE PHYSICAL EDUCATION AND SPORT}

\section{JULY 2ND-7TH, 1994}

\section{Prague, Czech RePublic}

The 9th Biennial Conference will be held at the Faculty of Physical Education and Sports at the Charles University. The scientific programme of the Conference will deal especially with the questions of seeking the best ways to attract people to physical activity engagement and to compare approaches to exercise in difference parts of the world.

ISCPES Conferences provide opportunities to share ideas and research, to stimulate and enthuse, and to reflect on principles, policies and practices in physical education, sport and related activity in a variety of national and cross-cultural contexts.

Main topics will be:

- Physical Activity for Life: Occidental/Oriental Comparisons

- Physical Activity in Developed and Developing Countries: A Comparative Focus

- New Concepts in the Training of Physical Education Teachers and Coaches

- Comparative and International Studies in Physical Education and Sport Curricula

- Physical Activity for Special Populations (e.g. Physically Challenged, Ethnic, Gifted etc.)

- Physical Activity in Health Education in Comparative Perspective

For further information, please contact: Conference Secretariat, 9th Biennial Conference of ISCPES, Ass. Prof. PhDr. Václav Velebil, FTVS UK, Martiho 31, 16252 Praha 6, Czech Republic. Tel: 004223372159 or Fax: 00422364075. 